\title{
The need for academic middleware to support advanced learning services
}

\author{
M. Konstantopoulos ${ }^{\mathrm{a}, \mathrm{b}, *}$, T. Spyrou ${ }^{\mathrm{a}, \mathrm{b}}$, J. Darzentas ${ }^{\mathrm{a}}$ \\ a Department of Product and Systems Design, University of the Aegean, GR 84100 Hermoupolis, Syros, Greece \\ ${ }^{\mathrm{b}}$ Department of Information and Communication Systems, University of the Aegean, GR 83200 Samos, Greece
}

\begin{abstract}
The increased significance of digital and human networks in academic institutions and the development of vocational and distance-learning methods and systems and their associated delivery services have modified the organizational and information structures of academic institutions. In this new scenario, management information systems, many learning resources in printed or digital format and network-related user services compete for acceptance in a rapidly changing system. There is a growing need to provide new services in order to function in a changing international market, to prepare for the expected student mobility and the increasing diversity of educational needs. There are two main research areas that are looking at the problem, each from their own perspective: the learning technology area and the network technology area. The former is working mainly on standards and best practices for the delivery of online and distance education. Efforts include the description of learning objects with metadata, the description of learners with profiles and the generic depiction of learning technology systems in terms of information systems architecture. In the latter research area, recent deployment issues have been of increasing concern. Inevitably, research is moving towards an area where major work is already underway. This is the part of the networking community that is working on the definition and deployment of middleware services. This paper argues for the introduction of academic middleware to speed up the delivery process of the new services and reduce the complexity of the managed systems. (c) 2001 Elsevier Science B.V. All rights reserved.
\end{abstract}

Keywords: Middleware; Learning technology; User profiles; Learning objects; Directory services

\section{Introduction}

Technology and economy are the two main driving forces behind the acceleration of the transformation of present day online education delivery. Technology enables the delivery of new types

\footnotetext{
${ }^{*}$ Corresponding author.

E-mail addresses: mpk@aegean.gr (M. Konstantopoulos), tsp@aegean.gr (T. Spyrou), idarz@aegean.gr (J. Darzentas).
}

of educational services. Computer networks are the main tools for the introduction of these new services. The most basic contribution of networks is the rapid transformation of education from location-based to distance-online-based [20]. This affects the roles of both the participants and the organizations involved. New entities enter the education arena such as information technology experts and commercial producers of educational software. Economically, organizations are investing in human intellectual capital, and training organizations are discovering the "corporate learning 
industry' [8]. The need for life-long vocational training, the growing trend for student exchange and the on growing demand for interdisciplinary studies, are all parts of the move away from traditional education based on either the acquisition of a degree or the binding of the student to a specific institution. The promotion of autonomy has become accepted as a goal in the education of children and adolescents [23] and network technology, with its benefits and disadvantages, [29] promotes this tendency.

Organizations are rethinking their modus operandi based on these facts. Popular courses are offered under franchise to other institutions [1], a growing number of institutions are participating in student exchange programs [10,27], and the introduction of distance-learning programs is a common policy both for academic institutions and other learning organizations. A number of strategies are being called into play within this online environment. Geographical constraints are lifted, and increasingly, the emphasis is upon individualized courses of learning rather than standardized offerings from institutions. This is particularly true in training organizations [28]. For instance, programmes of study are being offered that are modular in construction, with increasing flexibility as to how students combine modules within a discipline, or select modules across disciplines.

Clearly the most important new element that has arisen is inter-institutional communication. Student exchange, learning modules and franchise and inter-institutional degrees necessitate systems that will facilitate communication [11]. Apart from the alleged need for integrated information systems to support the institutional organizational and educational components (user network services, learning technology systems, institutional management information systems), there is an increasing need for any such system to be scalable and adaptable to a complex and globally expanding environment [4].

There are two main research areas that are looking at the problem, each from its own perspective: the learning technology area and the network technology area. The former is working mainly on standards and best practices for the delivery of online and distance education. Efforts include the description of learning objects with metadata $[3,16,19]$, the description of learners with profiles [12] and the generic description of learning technology systems in terms of information systems architecture [13]. In the latter research area, recent deployment issues have been of increasing concern [14,24,25]. Inevitably, research is moving towards an area where major work is already underway; this is the part of the networking community that is working on the definition and deployment of middleware services.

The term middleware was initially used in a software engineering context [6], but currently it is mainly related to the network services deployment area, tying together networks and services. Moreover, the set up of middleware communities [http://www.internet2.edu/middleware, http://www. terena.nl/middleware], dealing with deployment issues of such services, indicates that middleware services are more complex than protocol definitions. While still no consensus can be reached on the services that must be considered to be "middleware", there are some that are a common denominator. Such services are commonplace in present day organizational information systems but more work is required on the definition of a common inter-organizational framework. While both these areas of research are moving quickly to standardize and implement their work, there seems to exist a gap in the establishment of a surrounding environment for the initiation and acceptance of these standardization efforts in the work place, in this case educational institutions and those dealing with education delivery.

This paper argues the need for the specification of middleware to support the new functions of the educational community. The next section presents the problem area and summarizes the requirements for the emerging educational services of the future. Section 3 gives an overview of the advancements in technology in the areas of the learning technology and networking middleware services. Section 4 sketches a future implementation based on the results of previous work in the field. Finally, conclusions and future directions are given in Section 5. 


\section{Education in a new setting}

The education sector is directly connected with the development of society. The provision of education cannot be considered outside this context. Summarizing the essential changes in the education sector, the following facts are beyond doubt:

- The economic landscape is rapidly changing. Apart from the privatization of a large portion of the education sector, there is a growing need for life-long learning.

- The "new economy" demands a highly educated work force with up-to-date knowledge [7]. This fact is changing the clientele-and consequently the programme - of educational institutions.

- The Internet is offering new tools for the delivery of life-long learning programs at substantially reduced costs [4].

- Competing educational institutions are increasingly requesting the provision of an integrated service for both traditional and distance learners with equally attractive programs of study with the possibility of swapping from location-based to distance-online based or to a modular programme of study. Finally, instruction materials are steadily becoming more reliant on the use of learning technology. Computer labs, networks, digital learning environments and systems are all considered indispensable tools for the delivery of knowledge.

The main application areas of the near future are believed to be:

- The introduction of distance-learning programs as part of the regular studies in an institution.

- The need for further modularization of the study programme in order to offer customized degrees and to enhance student autonomy.

- The development of inter-institutional programs.

The main technological infrastructures that exist in a typical institution presently are:

- One or more custom management information systems that contain student records, financial data, etc.
- One or more distance-learning programs.

- A campus network and associated user services.

Recent research indicates that, of the competing learning institutions in this area, the successful ones will be those that manage to offer new services, while satisfying quality criteria applicable to a number of processes and entities like course development, faculty training, student services, learning resources, infrastructure, and assessment of results. A set of benchmarks [27] to evaluate the quality of the distance-learning programs in the US was better satisfied by an integrated information system. For example, the benchmarks on "Student Support" and "Faculty Support" presuppose that the institution, as an organization with its infrastructure, is directly responsible for the delivery of the courses (e.g., "Electronic security measures are in place to ensure the integrity and validity of information" or "Support for building and maintaining the distance education infrastructure is addressed by a centralized system").

In this situation, where the complexity of the system steadily increases and where the networkbased, global, inter-institutional services are continuously gaining importance, the introduction of institutional middleware would:

- increase the modularity and expandability of the educational information systems,

- accelerate the adoption of student exchange programs and inter-institutional learning programs and,

- create a context for the incorporation of distance-based programs as part of the regular programmes of study.

\section{The technology: implementation and standardi- zation}

In the learning technology area, there are efforts aimed at the promotion of interoperability between the different learning systems and the management of pedagogic elements. Therefore, standardization is directed to those components that can be considered reusable and therefore possessing commercial value (like the learning objects) 
or those components that are essential for the provision of the new services (like the profiles of users/learners). While there is also mobility in other areas, like the generic learning technology architecture, the student identifiers, and the learning environments, the above-mentioned information (learner profiles and learning material) is considered central to the educational procedure [21].

The pedagogic elements (or learning materials) are the digital information content to be provided to the students. In this field, there are some very advanced efforts on the design of learning materials metadata. The most widespread of these are the IEEE's Learning Resource Metadata [17], Ariadne [3] and IMS [18,19]. These efforts have led the technology in the field and have produced metadata object schemes. The design of these specifications was based on the possible uses of the learning resources as part of learning systems. Noteworthy categories of the LOM scheme are the Educational category that "groups the educational and pedagogic features of the resource", and the Rights category that "groups the features that deal with the conditions of use for the resource" [16]. On the other hand, the learner profiles in IEEE are described by PAPI [12]. The PAPI specification divides learner information into four types:

1. Personal information, such as names, phone numbers, addresses, etc.

2. Preference information, such as whether the learner prefers video over just plain text.

3. Performance information, referring to the learners' achievements in the context of learning environments (digitized or not) and finally.

4. Portfolio information, referring to the record of student achievements in terms of skills acquired, and abilities.

On the networking side, the main research includes the provision of an intermediate service between the network and the application level, basically for assistance in the deployment of distributed applications. In this context, the main effort has been given to the development of infrastructure on directories and public key in- frastructure in order to support functionalities authentication, authorization, accounting (AAA), applications of Quality of Service (QoS), active networks, distributed data management, information sharing. On the application level, there are some significant efforts (like GRID [9]) that, while they support academic research and experiments, do not yet support education especially in its new context as earlier described. However, the development of a wide-scale, global directory, as a crucial component for the provision of any user related distributed service, affects the provision of education also. This is especially true now that technology is unifying operating systems, mail servers and Web server user records, and the trend is to expand these directories in order to store profiles and define access rights for as many applications as possible running in a digitized system $[5,26]$. The speed, at which technology is moving, points to a situation where student records would be some kind of operating system user records [22]. This appears most likely since the directory services protocol and corresponding implementations of it are moving in this direction, not just for educational organizations but for any kind of organization, and especially those that are multinational and globally distributed. It is now accepted that a well-established directory service offers a stable and tested environment for the purposes of storing and manipulating, among other things, records related to the provision of education.

Middleware is not just a simple collection of protocols. The notion of middleware also contains the provision of a service. Therefore, middleware is not considered to be a set of interfaces, but system components with functionality. Thanks to middleware technologies, these services have the ability to scale globally [31]. A definition states [2] "Middleware can be viewed as a reusable, expandable set of services and functions that are commonly needed by many applications to function well in a networked environment". The provision of educational services over or using the network can be considered a typical application.

Research is ongoing for the integration of learning environments with organizational middleware services. In this context, the GESTALT 
project produced a demonstrator of a resource discovery service (RDS) with retrieval mechanisms on metadata [15] combined with portable student records using a directory services model [30].

The objective of GESTALT was to design and implement an open online learning environment by bringing together and enhancing the results of previous projects and creating an integrated trial system, which encompasses the whole of the delivery of online learning from searching for learning courses and resources, enrolling in and following programs of study delivered over networks, and providing mechanisms for passing information between various parts of the system in a secure and trusted manner.

Fig. 1 summarizes the GESTALT functional architecture. From one point of view, central to such a system are the user profiles and the asset management system. These are the two main stores for the learner and educational content data. User profiles hold user preferences for interaction (e.g., preferred language) with the learning environment (LE), with the RDS and other information such as personal portfolio and performance data. The asset management system controls access to added value resources, for example, course/module data, which could be accessed as an extra cost program of study. This subsystem also publicizes learning objects (i.e,. course module data) to the RDS.

Although not shown in the figure, other components like the RDS, the LE and the administration component have or can have their own

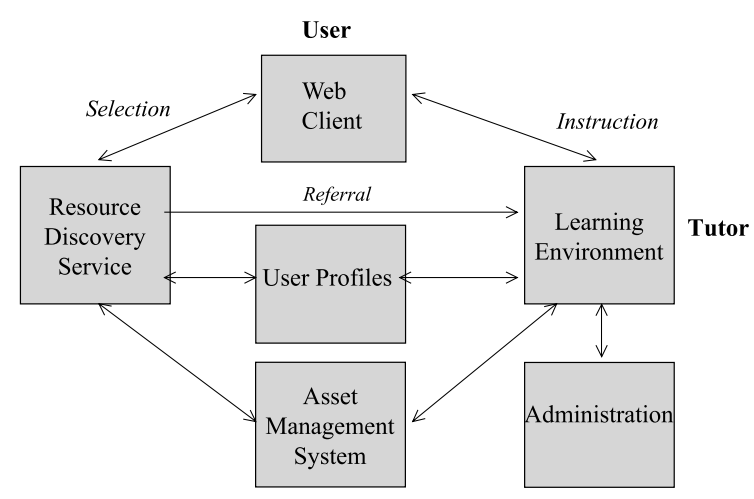

Fig. 1. The overall GESTALT functional architecture. directories holding information specific for their functions but not of direct interest to the other components. GESTALT indicated that in order for the various learning environments and brokerage services (like the RDS) to be capable of interacting - and to cooperate on the data exchange - which is crucial in order to add value to their services - the definition of common descriptions of the exchanged data is required. Moreover such descriptions must be integrated in the information systems of the related parties (e.g., academic institutions) and should be incorporated in a wider context, managing related chunks of information. This work has now been undertaken by a new project GUARDIANS (Gateway for User Access to Remote Distributed Information and Network Services).

\section{The vision}

The problem areas of the research presented in the previous section can be summarized as follows:

- There is an application area (Learning Technology area) whose deployment is basically based on the middleware infrastructure of the academic campuses and has not yet coordinated its activities with middleware services area in order to profit from middleware infrastructure.

- There is an underlying (middleware service) area having as a target the support of global distributed services but it has not yet considered the orientation of its activities to offer its widespread applications to the development of new educational methods and products for learners.

The development of an academic middleware based on the existing middleware efforts of the "networkers" and having learning technology as its objective would go quite some way towards resolving the problem and giving a boost to both areas. The vital questions are: What should such a middleware contain? And how should the middleware be constructed?

As Aiken et al. [2] noted, "the better approach would be to consider middleware as an 
unstructured, often orthogonal, collection of components (such as resources and services) that could be utilized either individually or in various subsets". Stretching this hypothesis, middleware is considered to be a set of components placed horizontally in the academic information infrastructure. Middleware is not a new product but rather a new service. Academic middleware, like all others, should contain the most common data, components or functions of the systems. This way this new service can become central to the intra-institutional information system. As was previously analysed the main information to be manipulated by such a component must be the learner (student, client) information and the module and learning content information from which the learning programs or qualifications or degrees are constructed (Fig. 2).

The problem of how the middleware should be constructed is directly related to the direction of the deployment of network middleware services. It is highly probable that directory services will play a central role. It is also a requirement that such middleware inherit implemented best practices of other middleware initiatives like the student identifiers, AAA and QoS schemes. The degree of interdependence between the two areas of research

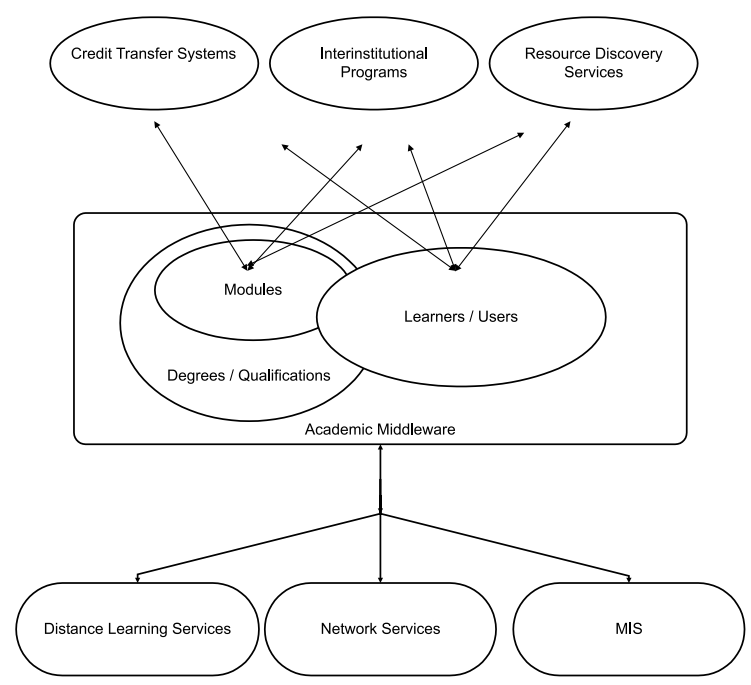

Fig. 2. Systems and services in connection with middleware. will depend upon the applications that will exploit this new framework.

The possible scenarios for use of the academic middleware are perhaps the best indication of the level at which academic middleware will assist the deployment of new academic services:

- Mixed location and distance-based programs. The students may have an option as to which path of study to follow. Based on the common student records serving both the MIS and the distance-learning environments, this need is addressed directly.

- Inter-institutional credit system. Agreements between institutions will be possible on credit exchange. The provision of inter-institutional degrees on a one-to-one basis or by federations of institutions is also possible. The definition of access rights to modules and programs can be easily configured, granted that the legal and further operational milieu are delineated.

- Provision of common middleware services for educational procedure. For example, distance based programs connected with learning technology environments with QoS and AAA directly applied.

As these new services illustrate, the development of middleware will affect both the communication of components in the intra-institutional and inter-institutional environment. This dual nature of middleware services requires that its deployment takes into account international standards and practice (Fig. 3).

\section{Conclusions}

The ideas discussed in this paper concern the implementation of middleware infrastructure with learning technology. Middleware as a notion is tied with network technology. It is expected that the significance of middleware services will increase correspondingly with the development of distributed, network-based services. Widely implemented middleware will greatly reduce the complexity of developing worldwide services. 


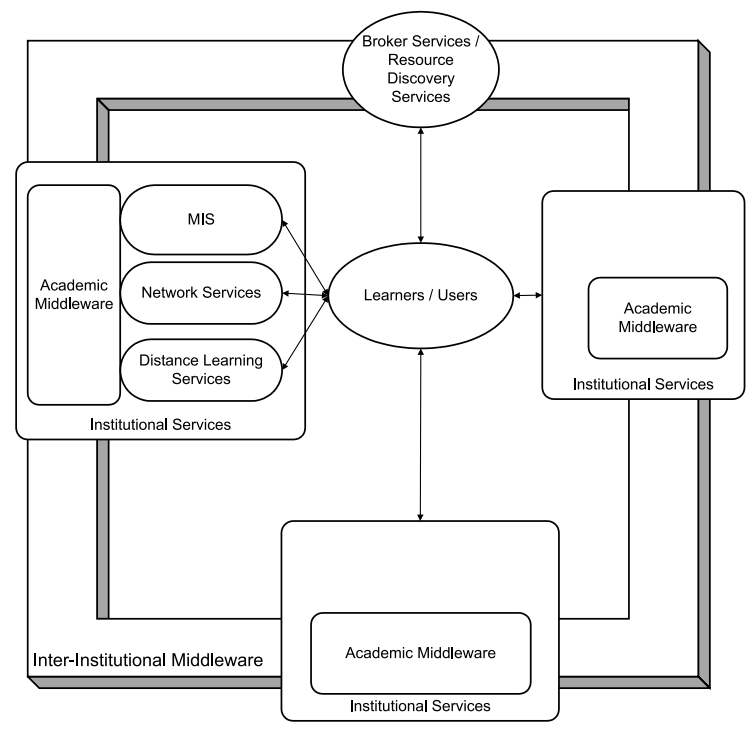

Fig. 3. Users and the inter-institutional middleware.

Education, and especially academic education is an application area where new technologies are oftenfirst introduced. Future development of services based on networks, using these either on the inter-institution relations or the institution to learner's relations is a need deriving from the economical and social environment. While the deployment of these services will require a wider consensus among the participants, the idea of this paper is the definition of a technical framework (as yet at a conceptual level) for supporting them.

Developing middleware including the learners and the learning materials may be the first step in an effort to release the learners from the restricting frontiers of single institutions and for reusability of the learning materials on a global scale. Of course, the significance of such middleware, like all network-related technologies will increase in parallel with its adoption by the related parties. However, efforts such as IMS in the US and Prometheus (http://www.prom.org) in Europe and their agreement to work together demonstrate the depth of commitment from both educationalists and educational service suppliers to implement commercially viable, interoperable systems.

\section{Acknowledgements}

This work has been partly supported by the European Union through the IST Programme (ACTS Project GUARDIANS Gateway for User Access to Remote Distributed Information and Network Services) and the GRNET (Greek Research Network) SA.

We would like to thank Mrs. Carol de Groot for her invaluable help in preparing this paper for publication.

\section{References}

[1] Achievements in Higher Education at East Birmingham College (September 1997), Retrieved February 2, 2000 from the World Wide Web: http://www.ebham.ac.uk/ college_info/achievements_profile/achieve_main.html.

[2] B. Aiken, J. Strassner, B. Carpenter, I. Foster, C. Lynch, J. Mambretti, R. Moore, B. Teitelbaum, Network Policy and Services: A Report of a Workshop on Middleware, Request for Comments 2768, February 2000.

[3] ARIADNE, Educational Metadata Recommendation, Version 3.0. (C) EPFL (Lausanne, Switzerland), KUL (Leuven, Belgium) and the ARIADNE project, 1999, Retrieved March 27, 2000 from the World Wide Web: http://ariadne.unil.ch/Metadata/ariadne_metadata_v3finall. htm.

[4] Bank of America Securities, The e-Bang Theory. Education Industry Overview, Bank of America Securities, Montgomery Division, September 1999.

[5] BED Web Team, Active Directory Architecture, White Paper, 1999, Retrieved 10 March 2000 from the World Wide Web: http://www.microsoft.com/WINDOWS2000/ library/howitworks/activedirectory/adarch.asp.

[6] P. Bernstain, Middleware: A model for distributed system services, Communications of the ACM 39 (2) (1996) 86-98.

[7] R. Close, R. Humphreys, B. Ruttenbur, e-Learning \& Knowledge Technologies, SunTrust Equitable Securities, March 2000.

[8] F. McCrea, R. K. Gay, R. Bacon, Riding The Big Wave. A White Paper on the B2B e*Learning Industry, January 18, 2000.

[9] H. Eertink, Y. Demchenko, Notes from European Middleware Workshop (EMW2000), Retrieved 10 October 2000 from the World Wide Web: http://www.terena.nl/ middleware/emw2000notes01.html.

[10] European Parliament and Council, 20 April 95, Decision no. 819/95/ec of the 14 March 1995, Establishing the Community action programme 'Socrates', Official Journal of the European Communities No. L 87/10. 
[11] European Commission, ECTS User Guide, 31.03.1998, Retrieved September 15, 2000 from the World Wide Web: http://europa.eu.int/comm/education/socrates/guide-en. doc.

[12] F. Farance, J. Schoening, Public and Private Information (PAPI) Specification, 1998, Retrieved February 2, 2000 from the World Wide Web: http://edutool.com/papi.

[13] F. Farance, Learning Technology Systems Architecture (LTSA) Specification Draft, 1999, Retrieved February 2, 2000 from the World Wide Web: http://edutool.com/ ltsa/.

[14] E. Forte, F. Haenni, K. Warkentyne, E. Duval, K. Cardinaels, E. Vervaet, K. Hendrikx, M. Wentland-Forte, F. Simillion, Semantic and pedagogic interoperability mechanisms in the Ariadne educational repository, ACM SIGMOD Record 28 (1) (1999) 20-25.

[15] P. Foster, M. Kraner, A. Graziano, D0401 Courseware Metadata design, 1999, Retrieved 4 April 3, 2000 from the World Wide Web: http://www.fdgroup.co.uk/gestalt/ D0401.pdf.

[16] GUARDIANS (Gateway for User Access to Remote Distributed Information and Network Services) Technical Annex. Information Societies Technology (IST) Programme, 2000.

[17] IEEE LTSC, Draft Standard for Learning Object Metadata. P1484.12/D4.0, 2000, 1, Retrieved March 27, 2000 from the World Wide Web: http://ltsc.ieee.org/doc/wg12/ LOM_WD4.htm.

[18] IMS, Learning Resource Meta-data Information Model, Version 1.0., 1999, Retrieved February 2, 2000 from the World Wide Web: http://www.imsproject.org/metadata/ mdinfo01.html.

[19] IMS, Meta-data Best Practice and Implementation Guide, Version 1, Educause, 1999.

[20] M. Konstantopoulos, T. Spyrou, P. Koutsabasis, J.S. Darzentas, C. Lambrinoudakis, J. Darzentas, The role of directory services in online education delivery: The GESTALT example, Int. J. Continuing Eng. Educ. Life-Long Learning 11 (1/2) (2001) 5-24.

[21] M. Konstantopoulos, J.S. Darzentas, T. Spyrou, P. Koutsabasis, J. Darzentas, Towards Integration of Learning Objects Metadata and Learner Profiles Design: Lessons Learnt from GESTALT, Interactive Learning Environments, 2001, in press.

[22] Microsoft 1999, Microsoft Windows Active Directory: An Introduction to the Next Generation Directory Services, White Paper, 1999.

[23] M.G. Moore, Learner Autonomy: The second dimension of independent learning, Convergence 5 (1972) 76-88.

[24] D. Rehak, Carnegie Mellon Online: System Architecture, 1997, Retrieved September 27, 2000 from the World Wide Web: http://online.web.cmu.edu/public/information/publications/architecture/index.html.

[25] B. Smith, Higher Education \& Enterprise Learning Management Systems, 2000, Retrieved September 27, 2000 from the World Wide Web: http://www.convergemag.com/ Publications/CNVGNov98/highered/highered.shtm.
[26] SUN ${ }^{\mathrm{TM}}$, The Solaris ${ }^{\mathrm{TM}}$ Operating Environment, Data Sheet, 2000, Retrieved March 30, 2000 from the World Wide Web: http://www.sun.com/software/solaris/ds/ds-sol8oe/.

[27] U. Teichler, F. Maiworm, The ERASMUS Experience Major Findings of the ERASMUS Evaluation Research Project. Office for Official Publications of the European Communities, 1996, Retrieved February 2, 2000 from the World Wide Web: http://europa.eu.int/en/comm/dg22/socrates/erasmus/erexpe.doc.

[28] The Institute For Higher Education Policy, Quality On The Line, benchmarks For Success In Internet-Based Distance Education, April 2000.

[29] C.-H. Tu, On-line learning migration: from social learning theory to social presence theory in a CMC environment, J Network Comput. Application 23 (2000) 27-37.

[30] V. Wade, K. Riley, B. Banks, P. Foster, N. Evans-Mudie, Y. Nicol, P. Doherty, Object (Interface) Specification, Work Package 5, The GESTALT project, final draft, 1999.

[31] J. Waldo, The End of Protocols or, Middleware for the New Millennium, Middleware 2000 Keynotes, 2000, Retrieved September 9, 2000 from the World Wide Web: http://www.research.ibm.com/Middleware2000/Keynotes/ keynotes.html.

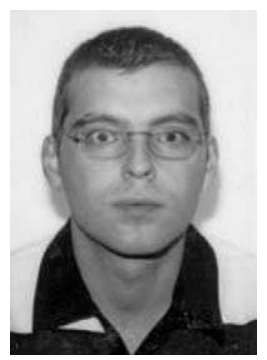

Michalis Konstantopoulos is the Technical Director of the GRNET directory service. $\mathrm{He}$ is a $\mathrm{Ph} . \mathrm{D}$. candidate in the Department of Information and Communication Systems, University of the Aegean, Greece. He has an M.Sc. and a Degree in Information Systems from the Department of Informatics, Athens University of Economics and Business, Greece. He has worked in several national and European projects and participated in the authoring of proposals, technical reports and research papers published in international conferences and journals and he has been technical director of the Greek national directory services program. $\mathrm{He}$ is currently working as a research associate for the "AegeanNet" network service and the GUARDIANS project.

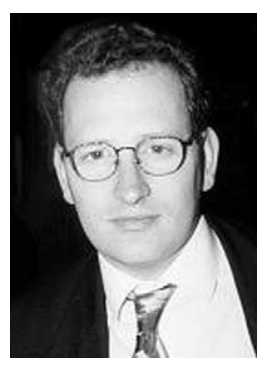

Thomas Spyrou is a Lecturer at the Department of Information and Communication Systems, University of the Aegean, Samos, Greece. He has participated to many research projects, funded nationally and by the European Union. He is a member of the Greek National Research Network (GRnet), the Greek Universities Network (GUnet) and Greek National Educational Network (EDUnet) technical committees. He is a member of the Organising Committee of the Department of Product and Systems Design, University of the Aegean, Syros, Greece. His current research interests include Intelligent Systems, Information Systems, Systems Thinking, Simulation modelling, Information Systems Security, Knowledge Management in Decision Aiding and Support Systems, Human-Computer Interaction Modelling, Intelligent agents and Distance and Life long Learning. 


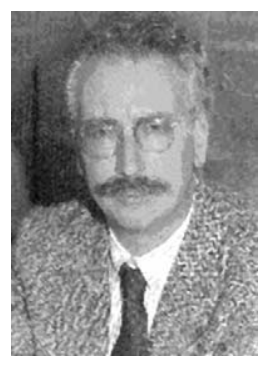

John Darzentas (M.Sc. Sussex, Ph.D.

London UK, Docent of the Abo Akademi, Finland) is the Acting Head of the Department of Product and Systems Design Information, University of the Aegean, Syros, Greece and Visiting Professor, University of Athens. He has collaborated in and led many research projects, both in the UK and Greece as well as projects funded by the European Community, on a range of subjects, including Systems Thinking; Decision Support; Simulation; Knowledge Management; Human Computer Interaction; Design; and Learning Technologies. He is author of a substantial number of papers in scientific journals and books. 